\title{
NOVEL PRINTED BOW-TIE ANTENNAS FOR DUAL-BAND DUAL-MODE MOBILE HANDSETS
}

\author{
Ágata Serrano-Vaello and David Sánchez-Hernández
}

\begin{abstract}
The recent developments of dual-band dual-mode D-AMPS/AMPS mobile handsets and radiostations has added pressure on GSM service providers to follow similar technology breakthroughs as a first step towards UMTS/FPLMTS. A frequency agile device accommodating both GSM standard frequency bands would serve more than 25 million GSM subscribers, with expectations as high as 100 millions subscribers by the year 2000. The complexity of the spectrum sharing between the two technologies and service generations may be alleviated by all terminal, system and network technologies. In this paper, several attempts to accomplish dual-band GSM/DCS 1800 operation on a single handset employing dual-band antenna concepts are introduced, and novel printed antenna elements developed at the Universidad Politécnica de Valencia are described.
\end{abstract}

\section{Introduction.}

The continuous increase of the Telecommunication market demand in recent years has consequently produced a rapid growth of mobile telephone networks as well as an extremely fast development of different mobile systems and technologies. The development of the American digital system D-AMPS with a dual standard providing, in the same network, analogue services (AMPS) at $800 \mathrm{MHz}$ and digital services (DAMPS) at $1900 \mathrm{MHz}$ has added pressure on GSM technology to get a fast migration towards third generation mobile communications systems UMTS/FPLMTS (more recently IMT2000). IMT2000 will integrate current mobile, wireless and satellite systems. In addition to voice, it will provide data and video transmission and multimedia services, and it will be compatible with fixed networks. For PCS operators, the dual-band concept as a first step in this migration bears important advantages. Networks operators can use the existing infrastructure at $800 \mathrm{MHz}$, whereas the spectrum at $1900 \mathrm{MHz}$ can be used to increase their capacity and attract new user categories to the networks [1]. This progressive migration of the second generation of GSM products (GSM, DCS 1800, DECT, PCS) towards third generation in Europe is expected to take form at the end of the millennium, enhancing therefore the attraction of new customers while ensuring that current users perceive an evolution of the system that is beneficial, attractive and natural.

This migration implies a necessary evolution of the current GSM system towards a single multiband multifunction handset allowing users to move freely between all existing cellular markets. Such a handset could be used by more than 25 million GSM subscribers in the world, with expectations as high as 100 millions subscribers by the year 2000 . The global phone, the international frequency-hopping wireless roamer, the one number, one caller I.D., one bill dream, appears to be the direction towards which the Telecommunications world is heading.

As a first step in the evolution towards UMTS, several solutions which guarantee the interoperativity of current systems are being investigated. The European Union has financed the ACTS projects to investigate new network, system and terminal technologies in order to alleviate the complexity of the spectrum sharing between the two current technologies and services generations. New Micro Base Stations (RBS 2301) for operators using GSM 900, DCS 1800 or PCS 1900 networks have already been introduced. To achieve this interoperativity, a great number of these investigations are concentrated on multiband antennas to allow users roaming any existing network and therefore, having a single handset to access a great number of services [2].

The authors are with the Departamento de Comunicaciones of the Universidad Politécnica de Valencia, Camino de Vera S/N, E-46071 Valencia, Spain. 


\section{The American AMPS/D-AMPS 1900 system.}

D-AMPS IS-136 technology is a digital standard which is based on TDMA standard in universal commercial service at $800 \mathrm{MHz}$. It is a dual standard which can provide in the same network analogue AMPS services and digital D-AMPS services. D-AMPS phones also operate in a dual-band mode at both 800 and $1900 \mathrm{MHz}$. A technique called "differentiated access" allows a single handset to serve as the subscriber's home phone, the company PBX extension at work, and a conventional mobile telephone for the user. D-AMPS IS-136 has achieved tremendous momentum in the United States with the active support of wireless network operators, vendors and others, and a it already has a fast growing global user base.

Thus, the American market has overtaken the European offering dual-band dual-mode handsets. There is even an adapter (HP 83206A) for the HP 8920B radio frequency communications test set which allows manufacturers of analogue/digital (dual mode) mobile phones compatible with TDMA IS-136 standards to completely characterise performance in seconds on the production line.

\section{The European GSM/DCS 1800 system.}

From the well known GSM standard at $900 \mathrm{MHz}$, two systems have been developed: DCS 1800 in the 1800 $\mathrm{MHz}$ band and PCS 1900 in the $1900 \mathrm{MHz}$ band, which use the same infrastructure and technology as GSM. In Europe, DCS 1800 complement the existing GSM networks while PCS 1900 is being introduced in the USA. DCS 1800 networks are currently operational, or being established in England, Germany, France, Switzerland and Austria in Europe, and in Thailand, Malaysia, Singapore, Chile Argentina and Hong Kong in the rest of the world, although many more countries are in process of issuing licenses for these networks. In Spain, Telefónica is planning to install a DCS 1800 pilot network in Barcelona. The advantages that a dual system GSM/DCS 1800 provides as a first step in the migration towards third generation are,

- A fast implantation in the market with reduced cost, because DCS 1800 uses the existing GSM infrastructure.

- Higher capacity, increased and focused coverage by means of the use of a cellular hierarchic structure.

- Higher voice quality.

- Higher binary rates.

- Differentiated service offers which allow the attraction of new kind of subscribers.

In accordance to this, several manufactures have announced the next introduction of dual-band GSM devices, but none of them are yet available in the Spanish market up to the time of writing.

\section{Antennas for mobile handsets.}

The monopole antenna has been the dominant radiating element in mobile phones up to now because of its great bandwidth, omnidirectional radiation pattern and low cost. Commercial dual handsets in USA use a structure consisting of two wire antennas: an helix and a monopole placed inside the helix.

Also in Europe, several configurations for dual terminals GSM/DCS 1800 are being studied. Two possibilities for realising dual frequency wire antennas have been developed in [3] as shown in figure 1. In the configuration of figure la, the helical antenna can be tuned to the lower frequency $(900 \mathrm{MHz})$ and the monopole placed inside to the higher frequency $(1800 \mathrm{MHz})$. Two helical antennas tuned to different frequencies can also be used as illustrated in figure $1 \mathrm{~b}$. Simulated and measured results suggested that the dual frequency operation with good matching at both frequencies could be obtained. Moreover, the measured bandwidths and radiation patterns are adequate for GSM/DCS 1800 system. An important drawback of these antennas is their great size, which is not desirable for small mobile terminals. This problem becomes more noticeable when diversity techniques are employed. 

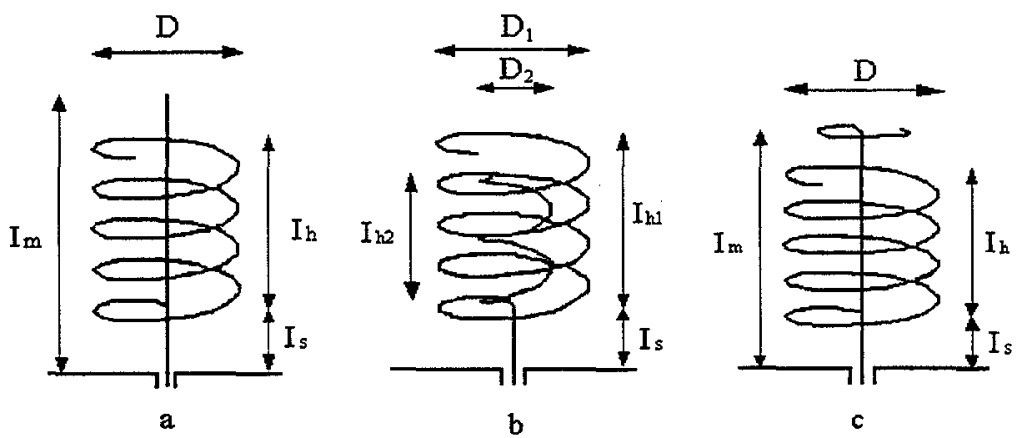

Figure 1. Dual frequency wire antenna configurations

a Monopole-helix

b Helix-helix

c Wound monopole-helix

To reduce the size of the antenna configuration, the dimensions of the helix can be decreased and the monopole can be wound according to figure 1c, but this solution also provides an insufficient bandwidth of the helical antenna, which has to be enlarged by means of a multi-resonant matching circuit.

Yet, the mobile handset requirements of small size, light weight, thin profile and low cost, bring up printed antennas as an interesting alternative for mobile terminals. In recent years, numerous research efforts have concentrated on printed elements which improve the radiation characteristics of the monopole. Namely, to reduce the high SAR (Specific Absorption Rate) in the users' head produced by a monopole antenna [4]. Studies on some of these printed elements include antennas like the PIFA (Planar Inverted F Antenna), BIFA (Back mounted Inverted F Antenna), RCDLA (Radiation-Coupled Dual-L Antenna), BMMDPA (Back Mounted Microstrip Double Patch Antenna) [5-6]. In fact, the first GSM mobile telephone with an integrated printed antenna FS-BIFA is now available in the market: the TCP-6000.

This dual band operation can also be obtained using multilayer structures, parasitic elements coupled to the main patch, log-periodic and quasi-log-periodic structures [7]. However, these structures haven't reached yet the commercial stage for mobile applications. This is mainly due to the fact that with most of these techniques, an increase in size or height of the antenna is expected, which is not desirable for mobile handset antennas where the conventional rectangular patch is already too big. It is then necessary to combine miniaturisation techniques with methods that allow a dual frequency operation without increasing the dimensions of the antenna.

Recently, a novel dual band PIFA for hand held portable telephones has been realised to operate at both 900 and $1800 \mathrm{MHz}$ bands [8]. The antenna consists of two separate radiation elements: a rectangular radiation element for operation at $1.8 \mathrm{GHz}$ and a L-shape radiation element as shown in figure 2. The dual band antenna is almost the same size as the single-band PIFA operating at $900 \mathrm{MHz}$.

Similarly, at the Departamento de Comunicaciones of the Universidad Politécnica de Valencia, several printed dual band antennas for dual band GSM/DCS 1800 systems have been developed. The techniques include a spur line filter embedded in the printed structure and a feed point optimisation [9]. It has already been demonstrated by the authors that the location of the feed point in microstrip antennas establishes and controls the trade-off between axial ratio and impedance bandwidth [10], an extremely important feature for mobile handset antennas.

\section{Dual-band bow-tie antenna.}

\subsection{Geometry of the antenna.}

The novel antenna consists of a bow-tie shape patch designed at $1.8 \mathrm{GHz}$ with a spur line filter embedded in the patch perimeter to achieve a new resonance at $925 \mathrm{MHz}$, as depicted in figure 3 . 


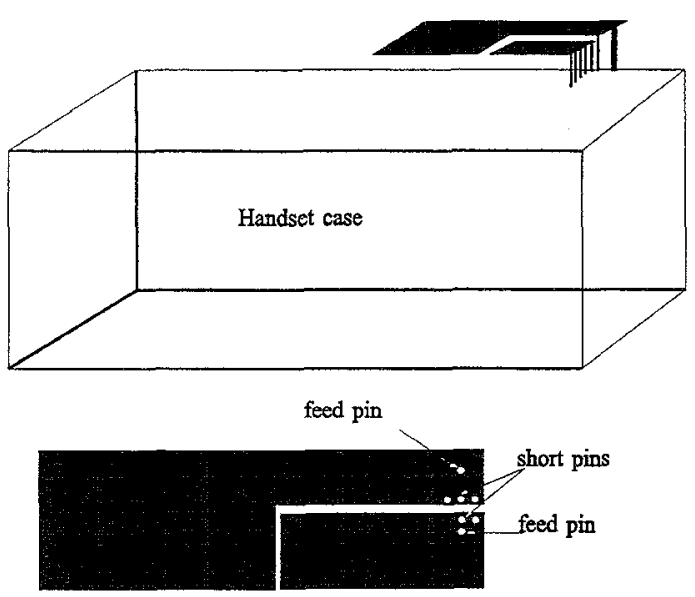

Figure 2. Dual band PIFA.

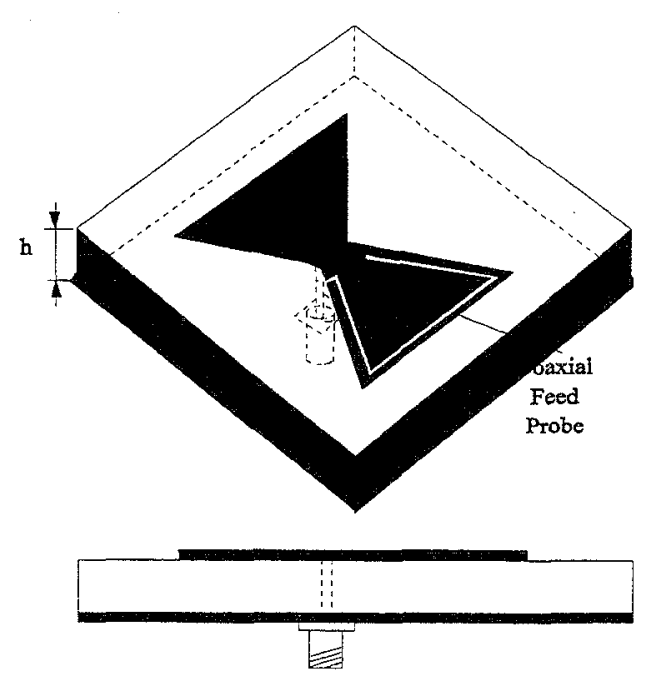

Figure 3. Dual band bow-tie antenna.

The patch is printed on a dielectric of thickness $\mathrm{h}=1.52 \mathrm{~mm}$ and relative permittivity $\varepsilon_{\mathrm{r}}=3.415$. The antenna is exited by a vertical SMA connector with the outer conductor connected to the ground plane of the antenna.

\section{A. The bow-tie patch.}

The resonant frequency of a printed antenna depends mainly on its length. Thus, lower resonant frequencies can be obtained increasing the length of the patch. To satisfy the small size requirement of mobile handset antennas, the patch has been designed at the higher operation frequency. The main advantage of the bow-tie patch is the reduction in size compared to a rectangular patch antenna designed at the same resonant frequency for the fundamental $\mathrm{TM}_{10}$ mode. The design formulas [11] show that the resonant frequency of the bow-tie antenna decreases with a decrease in the apex intersection $\left(W_{C}\right)$ and that, for a particular value of $W_{C}$, the resonant frequency also decreases with an increase in the irregular side length $L$.

\section{B. The spur-line filter.}

The idea behind the spur-line filter technique was to achieve a new resonance at $925 \mathrm{MHz}$ without increasing either the size or the thickness of the bow-tie antenna. Recent works [9] have shown that this is possible for a rectangular patch by using a spur-line filter within the width of the radiating edge of the patch opposite the feed point, forcing a modification of the currents and the generation of a new propagation mode with similar characteristics to the main mode. The dimensions of the filter have been calculated to obtain a centre frequency of $925 \mathrm{MHz}$ following the procedure described in [9]. The area reduction is astonishing: while an area of $534 \mathrm{~mm}^{2}$ is taken by the bow-tie patch resonating at both 925 and $1800 \mathrm{MHz}$, achieving a resonance at $925 \mathrm{MHz}$ with a conventional rectangular patch would need $12300 \mathrm{~mm}^{2}(24: 1)$. Besides, the radiation characteristics of both patches are similar.

\subsection{Simulated and measured results.}

The bandwidth of an antenna is defined by the VSWR and a value of VSWR $<2$ is a common standard as a range over which the bandwidth of microstrip patches can be evaluated. The VSWR of the bow-tie antenna was simulated using a 2.5D electromagnetic simulator and measured with a HP8720B network analyser. Simulated and measured results are shown in figures 4 to 6 . Initial measured bandwidths of $1.9 \%$ (17.63 $\mathrm{MHz}$ for $\left.\mathrm{f}_{1}=925 \mathrm{MHz}\right)$ and of $3.6 \%\left(64.17 \mathrm{MHz}\right.$ for $\left.\mathrm{f}_{2}=1.77 \mathrm{GHz}\right)$ are obtained, as shown in figure 4 .

The accurate location of the feed point plays a fundamental role in determining the bandwidth of the antenna for both bands. In accordance to this, an improvement in the bandwidth of each individual frequency band has been achieved, obtaining bandwidths of $2.4 \%\left(22.51 \mathrm{MHz}\right.$ for $\left.\mathrm{f}_{1}=925 \mathrm{MHz}\right)$ and $4 \%(70.47 \mathrm{MHz}$ for $\mathrm{f}_{2}=1.77 \mathrm{GHz}$ ), shown in figures 5 and 6 . It has to be said that these measured results do not satisfy the bandwidth requirements for GSM/DCS 1800 systems and they have to be further improved using multilayer structures, resonating circuits or a dual-fed bow-tie scheme. 


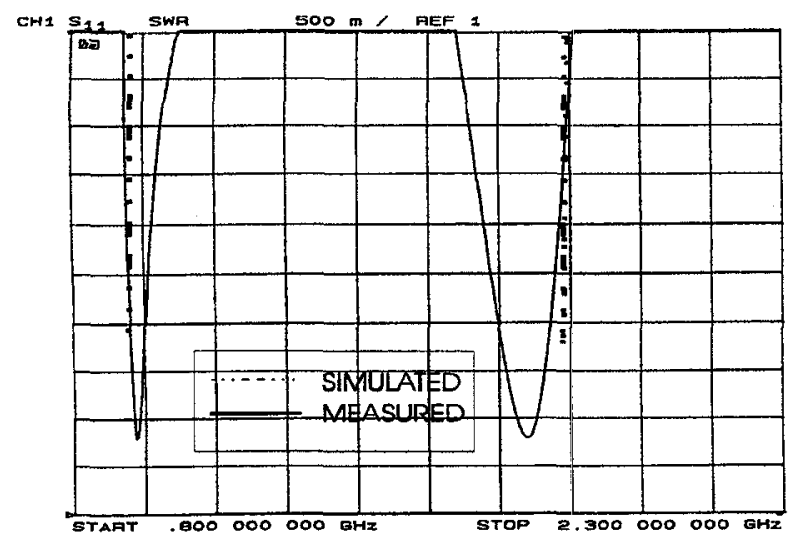

Figure 4. Simulated and measured VSWR of the new patch

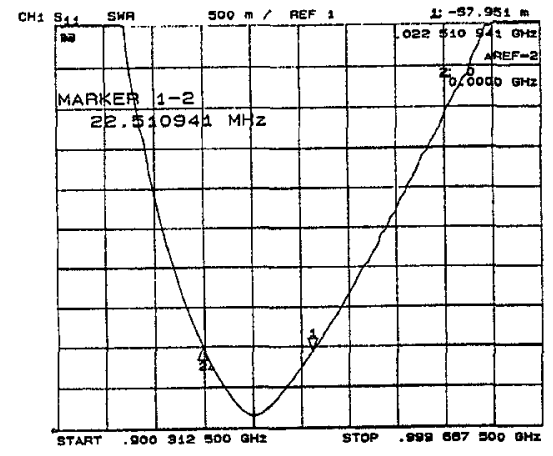

Figure 5. Measured VSWR for the first band.

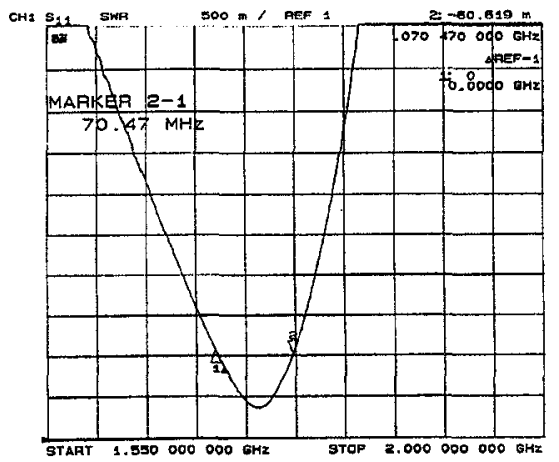

Figure 6. Measured VSWR for the second band.

The measured radiation patterns at the two different resonances are shown in figures 7 and 8 . The measured radiation patterns agreed well with the simulated ones and with those typical of patch antennas. This is also happening for single-band bow-tie patches as illustrated in figure 9 . The effect of the polarisation characteristics and position of the novel dual-band antenna integrated on a handheld terminal on SAR performance is currently under investigation. The numerical simulation for SAR estimation employs the Finite Integration Method. The FIT technique solves Maxwell's equations in integral form, and the analytical equations are discretised onto two orthogonal grids, yielding a set of discrete matrix equations. A sketch of the integrated antenna on a handset and its near electric field distribution can be observed in figure 10 where it can be compared to that of a monopole.

\section{Conclusions.}

The novel dual-band bow-tie antenna has shown excellent performance and an impressive size reduction compared to conventional patch antennas. The radiation characteristics of the novel antenna are similar to that of conventional microstrip patches. The achieved bandwidth, however, does not yet conform to the GSM/DCS 1800 bandwidth requirements, and dual-fed schemes where each band can be optimised individually appears as a promising way for increasing such bandwidth along with multilayer structures techniques.

Once the bandwidth problem is solved, the new structure has promising applications for future dual-band dual-mode systems worldwide. Future research include the determination of SAR performance for the new patch and further size reduction employing short circuit pins in a similar technique to that used in PIFA-like antennas.

\section{Acknowledgements.}

This work was supported by the Spanish Comision Interministerial de Ciencia y Tecnología, CICYT, under the TIC95-0983-C03-02 research project (Universidad Politécnica de Valencia). 


\section{References.}

[1] Isaksson, M. et al., 'D-AMPS 1900 - El sistema de comunicación personal de doble banda', Ericsson Review, 1995, no. 2, pp. 73-79. (in Spanish).

[2] Pourtaheri, A., 'GSM 900 / DCS 1800 - Soluciones Ericsson llave en mano para comunicaciones inalámbricas'. Ericsson Review, 1996, no. 2, pp. 48-57. (in Spanish).

[3] Eratuuli, P. et al., 'Dual frequency wire antennas', Electronics Letters, 1996, pp. 1051-1052.

[4] Jansen, M. et al.,'Interaction of handset antennas and a human in P.C.', Proceedings IEEE,1995, pp.7-17

[5] Shtrikman, I. et al., 'Performance and electromagnetic compatibility analysis of antennas for hand held mobile telephones', Proceedings of the 26th European Microwave Conference, 1996, pp. 246-250.

[6] Dlouhy, R. et al., 'Analysis of DCS- 1800 portable phones with microstrip antennas', Proceedings of the $26^{\text {th }}$ European Microwave Conference, 1996, pp. 242-245.

[7] Sánchez-Hernández, D. et al., 'A survey of broadband microstrip patch antennas', Microwave Journal, September 1996, pp. 67-84.

[8] Liu, Z.D. et al., 'Dual-band antenna for hand-held portable telephones', Electronics Letters, 1996, vol. 32 , no. 7 , pp. 609-610.

[9] Sánchez-Hernández, D. et al., 'Analysis and Design of a Dual Band Circularly Polarised Microstrip Patch Antenna', IEEE Transactions on Antennas and Propagation, 1995, Vol 43, No. 2, pp. 201-205.

[10] Sánchez-Hernández, D., et al., 'Dual-band Circularly Polarised Microstrip Antennas with a Single Feed', Electronics Letters, 1996, vol. 32, no. 25, pp. 2296-2298.

[11] George, J. et al., 'New compact microstrip antenna', Electronics Letters, 1996, pp. 508-509.

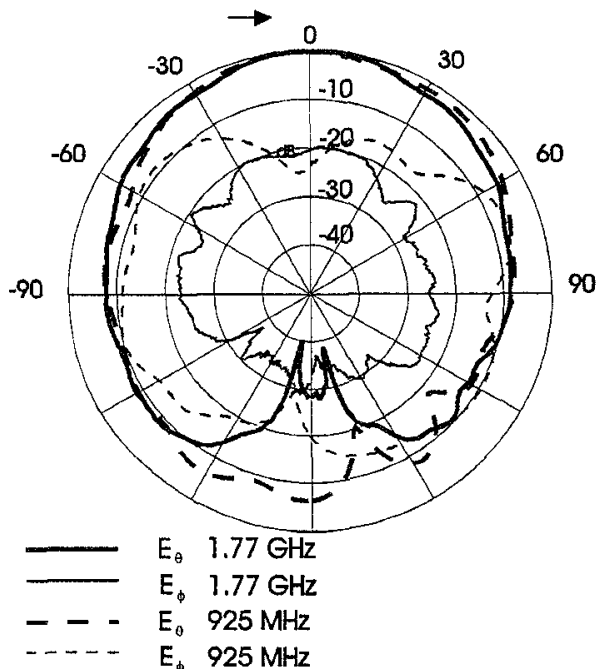

Figure 7. Measured E-plane radiation pattern for dual-band printed bow-tie antenna.

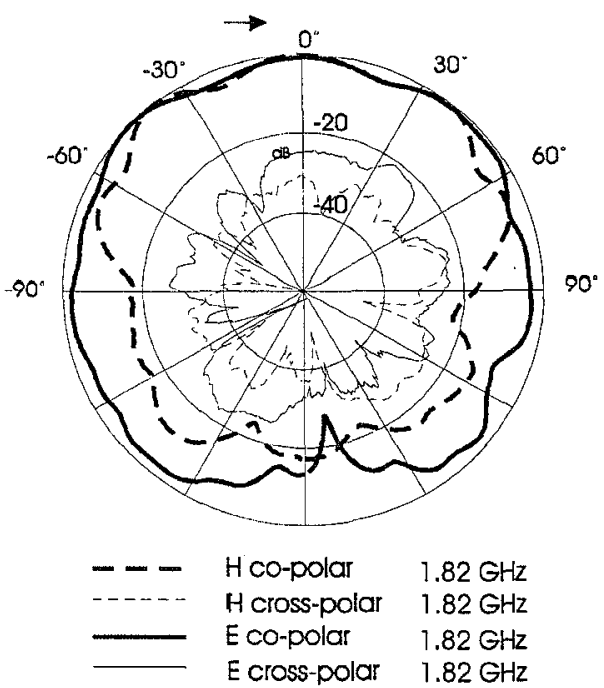

Figure 9. Measured E-plane radiation pattern for single-band bow-tie antenna at $1.82 \mathrm{GHz}$.

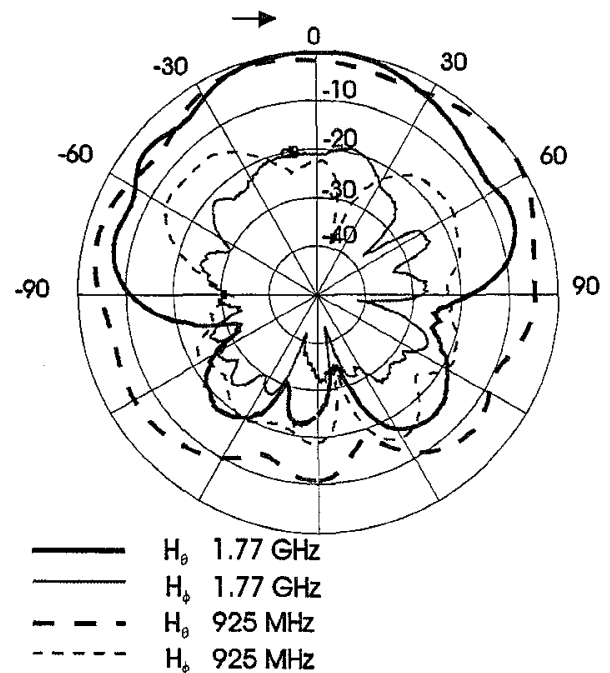

Figure 8. Measured H-plane radiation pattern for dual-band printed bow tie antenna.

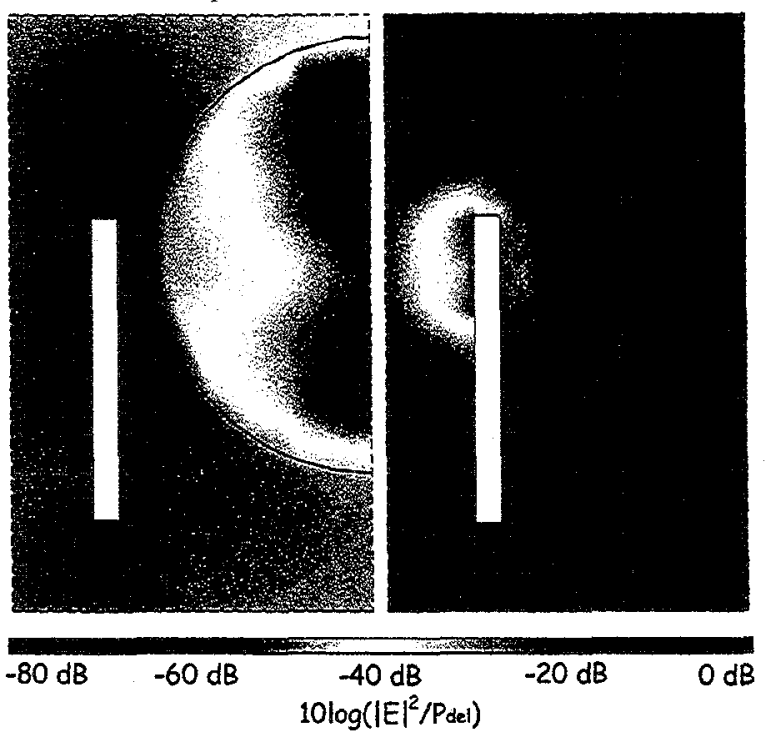

Figure 10. Computed normalised near field distribution at $1.8 \mathrm{GHz}$ for monopole (left and bow-tie antenna (right). 